\title{
0/1 Knapsack Problem using Diversity based Dual Population Genetic Algorithm
}

\author{
A. J. Umbarkar \\ Department of Information Technology, Walchand College of Engineering Sangli, MS, India \\ anantumbarkar@rediffmail.com \\ M. S. Joshi \\ Department of Computer Engineering, Jawaharlal Nehru Engineering College, Aurangabad, MS, India \\ madhuris.joshi@gmail.com
}

\begin{abstract}
The 0/1 Knapsack Problem is an optimization problem solved using various soft computing methods. The solution to the 0/1 Knapsack Problem (KP) can be viewed as the result of a sequence of decisions. Simple Genetic Algorithm (SGA) effectively solves knapsack problem for large data set. But it has problems like premature convergence and population diversity. Dual Population Genetic Algorithm (DPGA) is an improved version of Genetic Algorithm (GA) with the solution to above problems. This paper proposes Dual Population GA for solving 0/1 knapsack Problem. Experimental results of knapsack on SGA and DPGA are compared on standard as well as random data sets. The experimental result shows DPGA performs better than knapsack on SGA.
\end{abstract}

Index Terms- Dual Population Genetic Algorithm, DPGA, Genetic Algorithm, GA, Knapsack Problem, 0/1 knapsack problem, Evolutionary Algorithm, EA

\section{INTRODUCTION}

Genetic Algorithm (GA) is an optimization algorithm simpler than Calculus based optimization techniques and Dynamic Programming (DP) in implementation. It is used for solving problems in the area of industrial design by parameterization, Scheduling, Time Series Prediction, Single Machine Maximum Lateness Problem etc. [2]. But the problem associated with GA is that it is a population based algorith $m$ and as the populations evolve, they lose diversity and their individuals are trapped in local optima, especially when involving complex problems which have a lot of peaks in the fitness landscape [3].

GA is a stochastic optimization tool, it is a nondeterministic process. Therefore solution evolved using GA may or may not be optimized i.e. it doesn't ensure an optimal solution every time. This is called as premature convergence problem.

One of the solutions to premature convergence [4], [5] problem is population diversity based algorithm called Dual Population Genetic Algorithm (DPGA) [3], [6], [7], [18]. DPGA is a special type of GA where the two populations are generated viz. Main population and reserved population. The main population evolves similarly as to normal GA but reserve population has a special fitness function to provide diversity to the main population. Due to which all the search space is traversed and explored more effectively. This provides additional diversity to the main population. The information exchange between populations takes place through inter population crossbreeding. This technique helps to solve the problem of premature convergence [3], [6], [7].

0-1 knapsack problem (KP) is non deterministic polynomial (NP) problem. For NP problems, there are no precise and guaranteed algorithms that can run in polynomial time. Still it's possible to generate a solution and check it in polynomial time by predicting or guessing the solution. GAs can be used to solve the larger NP problem efficiently. GAs produce the possible solution set by approximation and randomization and try to find out the optimal solution which nearer to the exact solution in polynomial time.

This paper explains how DPGA is implemented for solving 0/1 Knapsack Problem. Section II contains a literature survey of how the GA is applied on 0/1 knapsack problem. Section III explains the DPGA and implementation procedure. Section IV explains how we implement 0/1 knapsack using DPGA. Section V gives the implementation details. Section VI comprises results and discussion. Finally, Section VII concludes the paper on the basis of results and statistical analysis.

\section{LITERATURE REVIEW}

Gordon and WimBOhm et.al. (1994) [8] proposed a note on the performance of GAs for Zero-One Knapsack Problems. In case of large problems branch-and-bound and depth-first methods outperform the GAs for finding optimal solutions and approximate solutions quickly. The results show the requirement for improved understanding of which problems are suitable for GAs and which problems are not.

Khuri, Back and Heitkotter (1994) [9] proposed the implementation of the 0/1 Multiple Knapsack Problem and GAs. In this work, GA allows for breeding and participation of infeasible strings in the population and allows in feasible breed strings to participate in the search as they do contribute information. Rather than augmenting the GA with domain-specific knowledge, introduced a fitness function with graded penalty term. 
Sakawa and Kato (2002) [10] proposed GAs with double strings for $0 / 1$ knapsack problem are first revisited together with some modifications and computational experiments. Then the GAs with double strings for $0 / 1$ knapsack problems are extended to deal with more general $0 / 1$ programming problems involving both positive and negative coefficients in the constraints. Especially, new decoding algorithms for double strings using reference solutions both without and with the reference solution updating procedure are proposed so that each of individuals is decoded to the corresponding feasible solution for the general $0 / 1$ programming problems. The efficiency and effectiveness of the proposed methods are investigated by comparing them with a branch and bound method with respect to the accuracy of an approximate solution and the processing time through a number of numerical experiments.

Hristakeva and Shrestha (2004) [11] applied GA to solve 0/1 KP problem. In the experimentation two selection methods, roulette-wheel and group selection improves the results quality with the elitism.

Julstrom (2005) [12] proposed greedy, genetic, and greedy GAs for the quadratic knapsack problem. The quadratic knapsack problem alters the traditional knapsack problem by assigning values to individual objects and to the pairs of objects. These results illustrate the power of evolutionary algorithms augmented with heuristic strategies to achieve good results on combinatorial problems.

Lin (2006) [13] experimented the KP with imprecise weight coefficients using GAs. Proposed approach simulates a fuzzy number by distributing it into some partition points. This work concluded that proposed fuzzy GA approach is different, and gives better results than the traditional fuzzy approach.

Shi (2006) [14] proposed the improved Ant Colony Algorithm (ACO) for 0/1 KP.

JoãoAlvesa, Almeidab (2007) [15] proposed a multi objective Tchebycheff based GA (MOTGA) for the multidimensional knapsack problem. This paper presented a new multi objective GA based on the Tchebycheff scalarizing function, which aims to generate a good approximation of the non dominated solution set of the multi objective problem. Computational results are presented and compared with the outcomes of other evolutionary algorithms. The number of potentially non dominated solutions obtained for each instance was not very high (namely when compared with MOGLS), but the solutions seem to be well dispersed and show good quality indicators.

Rohlfshagenand, Bullinaria (2007) [16] implemented an improved Exonic GA (ExGA II) for the Multiple Knapsack Problem. The algorithm's overall performance indicates good scaling properties, although further testing on larger problem sets is required to fully validate this claim.

Zhao, Man, Qi (2008) [17] proposed CGS-MSM PGA based on Multi-Agent and its application in solving 0-1 Knapsack Problem. After testing the algorithm through the simulation testing results show that the computational speed and precision are better than the traditional GA and the original CGS-MSM PGA.

Mohanty and Satapathy (2009) [18] proposed an evolutionary multi objective GA to solve 0/1 Knapsack Problem. Few numerical experiments are realized using the best and recent algorith $\mathrm{m}$ in this paper. In this paper, few numerical experiments are realized using the best and recent algorithm. The results show that the new proposed algorithm performs better than the existing evolutionary approach to this problem.

Zhao and Huang (2009) [19] implemented GA based on greedy strategy in the $0 / 1$ Knapsack Problem. In this paper, based on $0 / 1$ knapsack problem is given a mathematical model, and analysis of the greedy strategy. Given a GA to solve the knapsack problem, greedy strategy combining the traditional GA has been improved and shortened the time to solve, and to improve the accuracy of the solution. The improvement of this GA lies in the establishment of the original population, to use of greedy strategy solution as the original code of the algorithm.

Ahmed and Younas (2011) [20] proposed dynamic programming based GA to solve Multiple Knapsack Problems (MKP) problem. Proposed GA achieves the optimal solution and reduced the computation time.

Singh (2011) [21] applied the GA to solve the specific $0 / 1 \mathrm{KP}$ problem and experimented various selection method of GA.

Tuo, Yong, and Deng (2014) [22] proposed harmony search algorithm based on teaching-learning strategies (HSTL) for 0-1 KP.

Many researchers solved the $0 / 1 \mathrm{KP}$ by various methods with improvements in method or experimentation of variation in operators of method or comparing the performance operators of methods to solve KP. Most researchers' interest is in solving the KP problems with the new method or some improvements in the algorith $m$ rather than improving the performance of solving KP. The solution of KP by various methods should be compared with respect to efficiency, efficacy and success rate.

\section{DUAl POPUlAtion GENETIC AlgORITHM}

DPGA can be viewed as type of multi population GA in that it manipulates two isolated populations, but can also be considered a memory based algorith m because the additional population only plays the role of a repository for maintaining diversity or additional information [23].

For the main population to exploit the diversity preserved in the reserve population there must be means to exchange information between the populations. An individual of one population of a DPGA is unlikely to survive in the other because it gets contradictory fitness evaluations in different populations. A good individual in the main population, for example, may not look good in the reserve population because it does not contribute much to diversity. Therefore, DPGAs use interpopulation crossbreeding as a means of information exchange. 
DPGA starts with two randomly generated populations. The individuals of each population are evaluated by its own fitness function based on its evolutionary objective. The evolution of each population and the communication between populations is performed by inbreeding between parents from the same population, cross breeding between parents from different populations, and survival selection among these offspring [24]. Fitness functions for the reserve and main populations, procedure of reproduction and survival selection for both the populations are described as follows.

\section{A. Fitness Function for Main Population}

The main populations and reserve population of DPGA have different evolutionary objectives. Therefore they use different fitness functions. The fitness function $\mathbf{f m}$ of the main population is the objective function of the given problem because its objective is to find a good solution to the given problem.

\section{B. Fitness Function for Reserve Population}

Equation (1) is the fitness function of the reserve population, which gives a high fitness value to an individual to maintain an appropriate distance to the individuals in the main population thereby providing diversity to the main population.

$$
f r_{\delta}(x)=1-|\delta-d(M, x)|
$$

Where,

$d(M, x)$ : average distance $(0 \leq d(M, x) \leq 1)$ between the main population $\mathrm{M}$ and individual $x$ of the reserve population.

$\delta$ : desired distance $(0 \leq \delta \leq 1)$ between two population.

Assuming a binary representation for a chromosome $d(M, x)$ is calculated using equation (2) $[23,24,25]$.

\section{DPGA Algorithm}

DPGA [18, 19] steps for production of new generations are given in figure 1.

$$
\begin{aligned}
d(M, x) & =\frac{1}{|M|} \sum_{m \in M} h d(m, x) \\
& =\frac{1}{l} \sum_{k=1}^{l}\left|f_{M, k}-x_{k}\right|
\end{aligned}
$$

Where,

$m_{i}: i^{\text {th }}$ chromosome of the main population.

$h d\left(m_{i, x}\right)$ : Hamming distance between two chromosome vectors $m$ and $x$.

$l$ : length of chromosome.

$m_{i, k}: k^{\text {th }}$ gene on chromosome $m_{i}$ and $x_{k}$

$f_{M, k}$ : frequency of the $k^{\text {th }}$ gene value ' 1 ' of the main population $\mathrm{M}$.

$x_{i}$ : frequency of $k^{\text {th }}$ gene value ' 1 ' of the chromosome vector $\mathrm{x}$ and is identical to the $k^{\text {th }}$ gene value of the chromosome.

\section{KNAPSACK PROBLEM}

Knapsack problem is a constraint optimization problem. In this problem, a number of items $\mathrm{n}$ are provided. A knapsack with a certain capacity or volume $\mathrm{V}$ is provided. Each object $i$ has a specific positive integer volu me $V_{i}$ and a positive integer benefit $B_{i}$. Also there are $Q_{i}$ copies of the object $i$ such that $0 \leq Q_{i} \leq \infty$ available. In the knapsack problem, a combination of these items is to be selected so as to maximize the benefit but within the capacity of the sack.

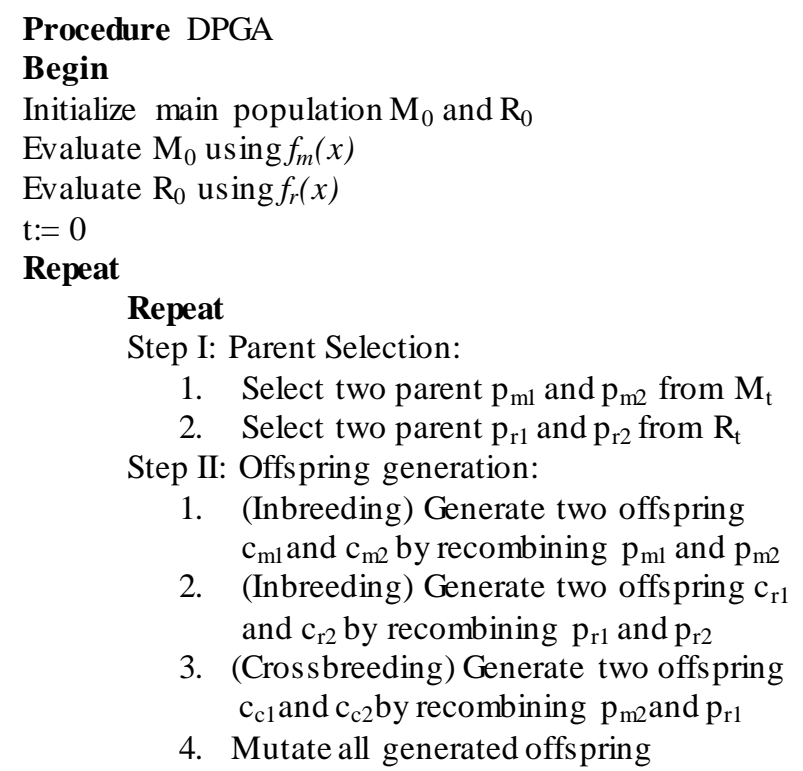

1. Select two parent $\mathrm{p}_{\mathrm{m} 1}$ and $\mathrm{p}_{\mathrm{m} 2}$ from $\mathrm{M}_{\mathrm{t}}$

2. Select two parent $\mathrm{p}_{\mathrm{r} 1}$ and $\mathrm{p}_{\mathrm{r} 2}$ from $\mathrm{R}_{\mathrm{t}}$

Step II: Offspring generation:

1. (Inbreeding) Generate two offspring $\mathrm{c}_{\mathrm{m} 1}$ and $\mathrm{c}_{\mathrm{m} 2}$ by recombining $\mathrm{p}_{\mathrm{m} 1}$ and $\mathrm{p}_{\mathrm{m} 2}$

2. (Inbreeding) Generate two offspring $c_{\mathrm{r} 1}$ and $\mathrm{c}_{\mathrm{r} 2}$ by recombining $\mathrm{p}_{\mathrm{r} 1}$ and $\mathrm{p}_{\mathrm{r} 2}$

3. (Crossbreeding) Generate two offspring $\mathrm{c}_{\mathrm{c} 1}$ and $\mathrm{c}_{\mathrm{c} 2}$ by recombining $\mathrm{p}_{\mathrm{m} 2}$ and $\mathrm{p}_{\mathrm{r} 1}$

4. Mutate all generated offspring

Step III: Evaluation \& Survival selection:

1. Evaluate $\mathrm{c}_{\mathrm{m} 1}, \mathrm{c}_{\mathrm{m} 2} \mathrm{c}_{\mathrm{c} 1}$ and $\mathrm{c}_{\mathrm{c} 2}$ i.e. $\mathrm{O}_{\mathrm{M}}$ using $f_{m}(x)$ and add the best two to $\mathbf{M}_{\mathbf{t}+1}$

2. Evaluate $\mathrm{c}_{\mathrm{r} 1}, \mathrm{c}_{\mathrm{r} 2}, \mathrm{c}_{\mathrm{c} 1}$ and $\mathrm{c}_{\mathrm{c} 2}$ i.e. $\mathrm{O}_{\mathrm{R}} \mathrm{using}$ $f_{r}(x)$ and add the best two to $\mathrm{R}_{\mathrm{t}+1}$

$$
\text { Until }\left|\mathrm{M}_{\mathrm{t}+1}\right|=\text { = pop_size }
$$

Until terminated $=$ true $\quad / /$ e.g., $t>t_{\max }$ End

Where,

$\mathrm{M}_{\mathrm{t}}$ and $\mathrm{R}_{\mathrm{t}}$ are main and reserve populations respectively.

$\mathrm{p}_{\mathrm{m} 1}$ and $\mathrm{p}_{\mathrm{m} 2}$ are selected parents from main population.

$\mathrm{p}_{\mathrm{r} 1}$ and $\mathrm{p}_{\mathrm{r} 2}$ are selected parents from reserve population.

$\mathrm{c}_{\mathrm{m} 1}$ and $\mathrm{c}_{\mathrm{m} 2}$ are child's of main population inbreeding. $\mathrm{c}_{\mathrm{c} 1}$ and $\mathrm{c}_{\mathrm{c} 1}$ are child's of cross sreeding.

$\mathrm{c}_{\mathrm{r} 1}$ and $\mathrm{c}_{\mathrm{r} 1}$ are child's of reserve population inbreeding.

$\mathrm{M}_{\mathrm{t}+1}$ and $\mathrm{R}_{\mathrm{t}+1}$ are next generation main and reserve populations respectively.

Fig. 1. Pseudo code of DPGA [24]

Let $X_{i}$ determine the number of copies of an object $i$ to be placed in the knapsack. And the objective function is to-

Maximize Benefit: $\sum_{\mathrm{i}=0}^{\mathrm{n}} \mathrm{B}_{\mathrm{i}} X_{i}$; 
Subject To Constraints: $\sum_{i=0}^{n} V_{i} X_{i} \leq V$

\section{IMPLEMENT ATION DET AILS OF 0-1 KP USING FOR GAS}

\section{A. Representation of the knapsack items}

Array is used as a data structure, to store benefits and volumes of items separately, as shown below:

Volumes:

\begin{tabular}{lllll}
1 & 2 & 3 & 4 & 5 \\
10 & 20 & 30 & 40 & 50 \\
\multicolumn{7}{l}{ Benefits: } \\
1 & 2 & 3 & 4 & 5 \\
5 & 10 & 15 & 20 & 25
\end{tabular}

The above diagram shows that item no. 1 has 10 as the volume and 5 as its benefit. Similarly, item no. 2 has 20 as its volume and 10 as its benefit and so on.

\section{B. Encoding of the chromosomes for knapsack problem}

An array is represented as a chromosome, having a size equal to the number of items in the sack (for example size is 5). Each element of an array denotes that whether an item is included in the sack means ' 1 ' or not in sack means ' 0 '. For example, the following chromosome indicates that the $1^{\text {st }}, 2^{\text {nd }}$ and 4 th item is included in the knapsack.

\begin{tabular}{lllll}
\multicolumn{2}{l}{ Encoding: } & & & \\
1 & 2 & 3 & 4 & 5 \\
1 & 1 & 0 & 1 & 0
\end{tabular}

A three-dimensional array (chromosomes [Size] [number of items] is used to represent the whole population of chromosomes). Size means the number of chromosomes in a population. The second dimension of an array represents the number of items that may good to be included in the knapsack. The third dimension of an array is used to represent the total volume and benefits of that chromosome. The data structure representation is same as it is in [2].

\section{Termination conditions used}

The GA terminates at completion of the number of generations.

\section{Fitness calculation}

Fitness calculation of each chromosome is done by summation of the benefits of the items that are added in the knapsack; with making sure that the capacity of the knapsack is not exceeded. To check the validity solution, we check the volume of the chromosome is lesser than the capacity of the knapsack, if not then one of the bits in the chromosome whose value is ' 1 ' is inverted and the chromosome is checked again [1].

\section{E. Selection method used}

In the GA evolution process, we used the elitis $m$ in which the two of the fittest chromosomes are copied as it to the next population, so the best solutions found in old population will not be lost.

\section{F. Crossover method used}

One point crossover is used to evolve the population. Crossover probability is $80 \%$.

\section{G. Mutation method used}

The mutation probability in this work $20 \%$ of the population. Mutation method used is one bit mutation.

\section{RESULTS AND DISCUSSION}

DPGA for constrained optimization problems is experimented on Intel Core-i3 processor system with Windows -7 as an operating system. The source code is developed in Java. The experiments are conducted on $0 / 1$ knapsack problem data sets. First data set is generated randomly for experimentation and called as the random data set. Second and third data sets are standard data sets taken from the standard resource.

\section{A. Random Data Set (Data set 1)}

Results are taken using random data set. Random data is generated on our above specified machine. Random data set is generated using a random number generator. These randomly generated numbers include weight and benefit of every object. This data set is input to the algorith $\mathrm{m}$. Data set 1 are used to compare performance of SGA with DPGA.

Weights $=\{14,29,13,36,15,19,33,36,13,36,36$, $31,43,26,16,16,23,30,24,27\}$

Benefits $=\{19,19,11,11,11,4,11,15,18,14,2,15$, $9,14,9,3,7,14,8,6\}$

\section{B. Standard Data Set (Data set 2)}

The standard data set 2 are taken from [26] for experimentation of $0 / 1$ knapsack problem. Data set 2 are used to compare performance of SGA with DPGA.

Weights $=$

$\{135,139,149,150,156,163,173,184,192,201,210,214,221$, $229,240\}$

Benefits $=\{70,73,77,80,82,87,90,94,98,106,110,113,11$ $5,118,120\}$

\section{Standard Data Set (Data set 3)}

Standard data set 3 are taken from github [27] for experimentation of $0 / 1$ knapsack proble $\mathrm{m}$. Th is data set 3 are used to compare performance of SGA with DPGA.

Weights $=\{56,59,80,64,75,17\}$

Benefits $=\{50,50,64,46,50,5\}$

The experimentation is carried on three on different data sets. The results taken on three data sets are shown in table I, II and III respectively. The parameter setting for each experiment is also shown in the respective tables. Figures 2, 3 and 4 are benefit \& generation nu mber versus reading index, for three data sets respectively. Figures 2, 3 and 4 shows that, the DPGA take a lesser number of 
generations than SGA and produces better solutions for three data sets.

Table 1. Parameter Settings and Results for Random Data SET 1

\begin{tabular}{|c|c|c|c|c|}
\hline \multicolumn{5}{|c|}{ Parameter Settings for Random Data } \\
\hline \multirow[t]{2}{*}{ Dimension } & \multirow[t]{2}{*}{20} & Capacity & \multirow[t]{2}{*}{$\begin{array}{l}\text { Population } \\
\text { Size }\end{array}$} & \multirow[t]{2}{*}{20} \\
\hline & & 400 & & \\
\hline $\begin{array}{c}\text { Standard } \\
\text { Benefit } \\
\end{array}$ & 200 & & $\begin{array}{c}\text { Generation } \\
\text { No. }\end{array}$ & 50 \\
\hline \multicolumn{5}{|c|}{ Knapsack Benefit and Generation No. } \\
\hline Sr. No & $\begin{array}{c}\text { SGA } \\
\text { Gen. No. }\end{array}$ & $\begin{array}{c}\text { SGA } \\
\text { Benefit }\end{array}$ & $\begin{array}{c}\text { DPGA } \\
\text { Gen. No. }\end{array}$ & $\begin{array}{l}\text { DPGA } \\
\text { Benefit }\end{array}$ \\
\hline 1 & 28 & 179 & 20 & 200 \\
\hline 2 & 50 & 200 & 34 & 200 \\
\hline 3 & 45 & 197 & 43 & 197 \\
\hline 4 & 22 & 197 & 22 & 197 \\
\hline 5 & 49 & 187 & 30 & 187 \\
\hline 6 & 33 & 198 & 33 & 198 \\
\hline 7 & 44 & 190 & 32 & 190 \\
\hline 8 & 50 & 192 & 29 & 192 \\
\hline 9 & 46 & 193 & 21 & 193 \\
\hline 10 & 38 & 198 & 38 & 198 \\
\hline \multicolumn{5}{|c|}{ Statistical Analysis } \\
\hline Benefit & \multicolumn{2}{|c|}{ SGA } & \multicolumn{2}{|c|}{ DPGA } \\
\hline Mean & \multicolumn{2}{|c|}{193.10} & \multicolumn{2}{|c|}{195.20} \\
\hline $\mathrm{SD}$ & \multicolumn{2}{|c|}{6.4368} & \multicolumn{2}{|c|}{4.4422} \\
\hline SEM & \multicolumn{2}{|c|}{2.0355} & \multicolumn{2}{|c|}{1.4047} \\
\hline
\end{tabular}

Table 2. Parameter Settings and Results for Standard data set 2

\begin{tabular}{|c|c|c|c|c|}
\hline \multicolumn{5}{|c|}{ Parameter Settings for Standard Data } \\
\hline \multirow[t]{2}{*}{ Dimension } & \multirow[t]{2}{*}{15} & Capacity & \multirow{2}{*}{$\begin{array}{l}\text { Population } \\
\text { Size }\end{array}$} & \multirow[t]{2}{*}{20} \\
\hline & & 750 & & \\
\hline $\begin{array}{l}\text { Standard } \\
\text { Benefit }\end{array}$ & 395 & & $\begin{array}{c}\text { Generation } \\
\text { No. }\end{array}$ & 50 \\
\hline \multicolumn{5}{|c|}{ Knapsack Benefit and Generation No. } \\
\hline Sr. No & $\begin{array}{c}\text { SGA } \\
\text { Gen. No. }\end{array}$ & $\begin{array}{c}\text { SGA } \\
\text { Benefit }\end{array}$ & $\begin{array}{c}\text { DPGA } \\
\text { Gen. No. }\end{array}$ & $\begin{array}{l}\text { DPGA } \\
\text { Benefit }\end{array}$ \\
\hline 1 & 63 & 395 & 83 & 395 \\
\hline 2 & 95 & 395 & 181 & 394 \\
\hline 3 & 127 & 395 & 152 & 394 \\
\hline 4 & 54 & 394 & 2 & 395 \\
\hline 5 & 15 & 395 & 1 & 395 \\
\hline 6 & 12 & 395 & 46 & 394 \\
\hline 7 & 51 & 395 & 37 & 394 \\
\hline 8 & 46 & 393 & 19 & 395 \\
\hline 9 & 49 & 395 & 5 & 395 \\
\hline 10 & 42 & 395 & 15 & 395 \\
\hline \multicolumn{5}{|c|}{ Statistical Analysis } \\
\hline \multicolumn{2}{|c|}{ Benefit } & SGA & \multicolumn{2}{|c|}{ DPGA } \\
\hline \multicolumn{2}{|l|}{ Mean } & 394.70 & \multicolumn{2}{|c|}{396.60} \\
\hline \multicolumn{2}{|l|}{$\mathrm{SD}$} & 0.6749 & \multicolumn{2}{|c|}{0.5163} \\
\hline \multicolumn{2}{|l|}{ SEM } & 0.1509 & \multicolumn{2}{|c|}{0.1154} \\
\hline
\end{tabular}

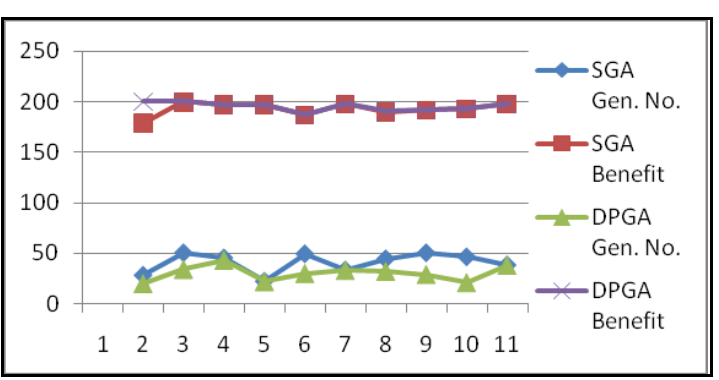

Fig. 2. Knapsack benefit \& generation number versus reading index for random data 1

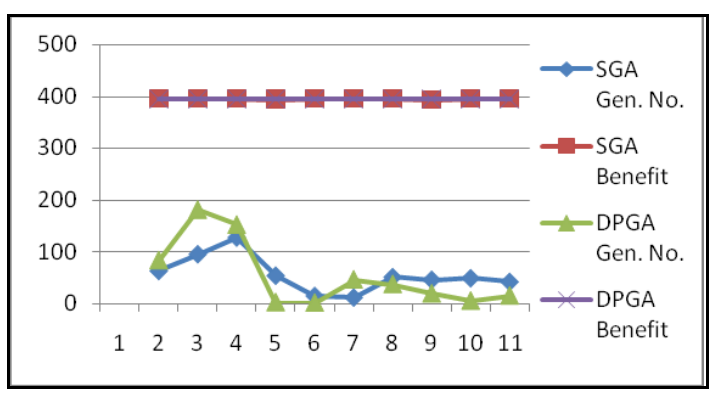

Fig. 3. Knapsack benefit \& generation number versus reading index for data set 2

Table 3. Parameter Settings and Results for Git data set 3

\begin{tabular}{|c|c|c|c|c|}
\hline \multicolumn{5}{|c|}{ Parameter Settings for Git Data } \\
\hline \multirow[t]{2}{*}{ Dimension } & \multirow[t]{2}{*}{6} & Capacity & \multirow{2}{*}{$\begin{array}{l}\text { Population } \\
\text { Size }\end{array}$} & \multirow[t]{2}{*}{10} \\
\hline & & 190 & & \\
\hline $\begin{array}{l}\text { Standard } \\
\text { Benefit }\end{array}$ & 150 & & $\begin{array}{c}\text { Generation } \\
\text { No. }\end{array}$ & 20 \\
\hline \multicolumn{5}{|c|}{ Knapsack Benefit and Generation No. } \\
\hline Sr. No & $\begin{array}{c}\text { SGA } \\
\text { Gen. No. }\end{array}$ & $\begin{array}{c}\text { SGA } \\
\text { Benefit }\end{array}$ & $\begin{array}{c}\text { DPGA } \\
\text { Gen. No. }\end{array}$ & $\begin{array}{l}\text { DPGA } \\
\text { Benefit }\end{array}$ \\
\hline 1 & 1 & 119 & 4 & 150 \\
\hline 2 & 1 & 150 & 1 & 150 \\
\hline 3 & 1 & 150 & 8 & 150 \\
\hline 4 & 10 & 150 & 1 & 146 \\
\hline 5 & 1 & 119 & 5 & 150 \\
\hline 6 & 19 & 146 & 1 & 150 \\
\hline 7 & 2 & 150 & 1 & 150 \\
\hline 8 & 2 & 150 & 1 & 150 \\
\hline 9 & 16 & 146 & 1 & 150 \\
\hline 10 & 15 & 146 & 1 & 150 \\
\hline \multicolumn{5}{|c|}{ Statistical Analysis } \\
\hline Benefit & \multicolumn{2}{|r|}{ SGA } & \multicolumn{2}{|c|}{ DPGA } \\
\hline Mean & \multicolumn{2}{|c|}{142.60} & \multicolumn{2}{|c|}{396.60} \\
\hline $\mathrm{SD}$ & \multicolumn{2}{|c|}{12.5715} & \multicolumn{2}{|c|}{1.2649} \\
\hline SEM & \multicolumn{2}{|c|}{3.9754} & \multicolumn{2}{|c|}{0.4000} \\
\hline
\end{tabular}

Table 4. Comparison of SGA and DPGA based on mean, standard deviation and standard error of mean for data sets.

\begin{tabular}{|c|c|c|c|c|c|c|}
\hline & \multicolumn{2}{|c|}{ Data set 1 } & \multicolumn{2}{c|}{ Data set 2 } & \multicolumn{2}{c|}{ Data set 3 } \\
\hline Benefit & SGA & DPGA & SGA & DPGA & SGA & DPGA \\
\hline M & 193.1 & 195.2 & 394.7 & 396.6 & 142.6 & 396.6 \\
\hline SD & 6.4 & 4.4 & 0.67 & 0.51 & 12.5 & 1.2 \\
\hline SEM & 2.0 & 1.4 & 0.15 & 0.11 & 3.9 & 0.40 \\
\hline
\end{tabular}

I.J. Intelligent Systems and Applications, 2014, 10, 34-40 


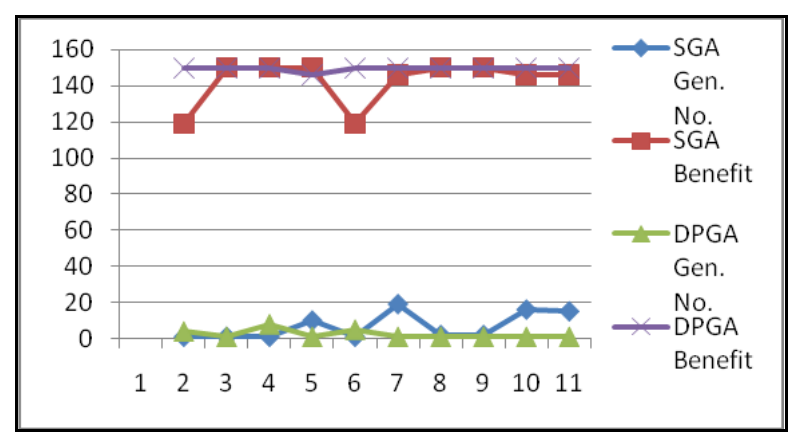

Fig. 4. Knapsack benefit \& generation number versus reading index for data set 3

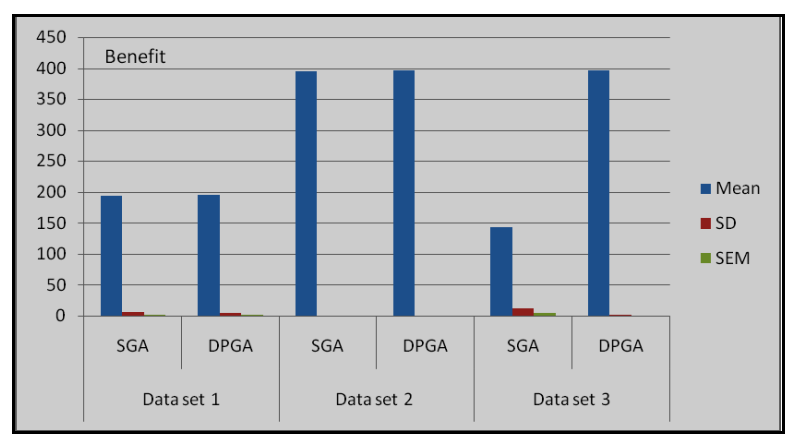

Fig. 5. Comparison of SGA and DPGA based on mean, standard deviation and standard error of mean for data sets.

Table IV shows the comparison of mean (M), standard deviation (SD) and standard error of mean (SEM). Values obtained for SD and SEM decrease in case of DPGA with the comparis on to SGA for the knapsack problem. Figure 5 shows the comparison of benefit given by SGA and DPGA based on mean, standard deviation and standard error of mean for data sets

From the experimental results, it is observed that the mean value obtained for knapsack benefit using DPGA is significantly better over the mean value obtained using SGA.

\section{CONCLUSION}

This paper presents a novel approach to solve $0 / 1$ knapsack problem using DPGA. DPGA provides a solution to problems like premature convergence and population diversity of SGA. Experimental results show that DPGA solves the 0/1 knapsack problem effectively than SGA. The additional population of DPGA provides population diversity by using the additional fitness function. Benefits achieved by $0 / 1$ knapsack problem are in lesser effort (function evaluation), with better efficacy (mean, standard deviation) and standard error of mean.

In future, the DPGA can also be checked for more complex knapsack problems. Further, DPGA can be improved for efficiency, efficacy and success rate by adding more reserve population to DPGA. A performance comparison of DPGA could be done with other metaheuristics.

\section{ACKNOWLEDGMENT}

The authors are thankful to the anonymous reviewers as well as the editors for their valuable comments and suggestions which have led to improve the quality of presentation of the paper. Our special thanks to T. Park, R. Choe and K.R. Ryu for their work on Dual Population Genetic Algorithm published in various journals and conferences.

\section{REFERENCES}

[1] Rahman K. and Ahmed S. Performance Analysis of Genetic Algorithm for Solving the Multiple-Choice MultiDimensional Knapsack Problem. International Journal of Recent Trends in Engineering, 2009, vol. 2, no. 2.

[2] http://www.informatics.indiana.edu/fil/CA S/PPT/Davis/sld 029.html, 12th August, 2013.

[3] Park T. and Ruy K. A Dual-Population Genetic Algorithm for Adaptive Diversity Control. IEEE transactions on evolutionary computation, December 2010, vol. 14 , no. 6 : 865-884.

[4] Ebrahimzadeh R. and Jampour M. Chaotic Genetic Algorithm based on Lorenz Chaotic System for Optimization Problems. International Journal of Intelligent Sy stems and Applications, 2013, Vol. 5( 5):19-24.

[5] Song, S., Kong L. and Cheng J. A Novel Particle Swarm Optimization Algorithm Model with Centroid and its Application. International Journal of Intelligent Systems and Applications, Vol.1 (1): 42-49.

[6] Umbarkar A. J. and Joshi M. S. Serial DPGA vs. Parallel Multithreaded DPGA: Threading Aspects. Proceedings of the International Conference on Soft Computing for Problem Solving (SocProS 2011), 2012: 39-50.

[7] Umbarkar A.J. and Joshi M.S. Dual Population Genetic Algorithm (GA) versus OpenMP GA for Multimodal Function Optimization. International Journal of Computer Applications, 2013, vol. 64: 29-36.

[8] Gordon V. and Wim BOhm A. P. A Note on the Performance of Genetic Algorithms on Zero-One Knapsack Problems. Proceeding of the 1994 ACM symposium on Applied computing, 1994: 194-195.

[9] Khuri S., Back T., and et.al. The Zero/One Multiple Knapsack Problem and Genetic Algorithms. Proceeding of the 1994 ACM symposium on Applied computing, 1994: 188-193.

[10] Sakawa M. and Kato K. Genetic algorithms with double strings for $0-1$ programming problems. European Journal of Operational Research, 2003, 144: 581-597.

[11] Hristakeva, M. and Shrestha, D. Solving the 0-1 knapsack problem with genetic algorithms. In Proceedings of the 37th Midwest Instruction and Computing Symposium, Morris, MN, Apr. 2004.

[12] Julstrom B. Genetic and Greedy Genetic Algorithms for the Quadratic Knapsack Problem. Proceeding of the 7th annual conference on Genetic and evolutionary computation, 2005: 607-614.

[13] Lin F. Solving the knapsack problem with imprecise weight coefficients using genetic algorithms. European Journal of Operational Research, 2008, 185: 133-145.

[14] Hanxiao, S. Solution to 0/1 Knapsack Problem Based on Improved Ant Colony, Algorithm. 2006 IEEE International Conference on Information Acquisition, 2006, 1062-1066.

[15] João Alves M., Marla. MOTGA: A multiobjective Tchebycheff based genetic algorithm for the 
multidimensional knapsack problem. Computers \& Operations Research, 2007, 34 (11): 3458-3470.

[16]Rohlfshagen P. ExGA II: An Improved Exonic Genetic Algorithm for the Multiple Knapsack Problems. Proceeding of the 9th annual conference on Genetic and evolutionary computation, 2007: 1357-1364.

[17] Zhao T. and Man Z. A CGS-MSM PGA based on MultiAgent and its application in solving 0-1 Knapsack Problem. Proceeding of First International Conference on Intelligent Networks and Intelligent Systems, 2008. ICINIS '08, 2008: $73-76$.

[18] Mohanty S. and Satapathy R. An evolutionary multiobjective genetic algorithm to solve 0/1 Knapsack Problem. Proceeding of 2nd IEEE International Conference on Computer Science and Information Technology, 2009. ICCSIT 2009: 397 - 399.

[19] Zhao J., Huang T., Pang F. and Liu Y. Genetic algorithm based on Greedy strategy in the 0-1 Knapsack Problem. Proceeding of 3rd International Conference on Genetic and Evolutionary Computing, 2009. WGEC '09, 2009: 105 107.

[20] Ahmed Z. and Younas I. A Dynamic Programming based GA for 0-1 Modified Knapsack Problem. International Journal of Computer Applications, 2011, 16(7):1-6.

[21] Singh, R.P. Solving 0-1 Knapsack problem using Genetic Algorithms. 2011 IEEE 3rd International Conference on Communication Software and Networks (ICCSN), 2011, 591-595.

[22] Tuo S., Yong L., and Deng F. A Novel Harmony Search Algorithm B ased on Teaching-Learning Strategies for 0-1 Knapsack Problems. The Scientific World Journal (2014) 2014, 1-19.

[23] Umbarkar A. J. and Joshi M. S. Review of parallel genetic algorithm based on computing paradigm and diversity in search space. ICTACT Journal on Soft Computing, 2013, vol. 3: 615-622.

[24] Park T. Choe R. and Ruy K. Adjusting Population Distance for the Dual-Population Genetic Algorithm. Proceedings of the 20th Australian joint conference on Advances in Artificial Intelligence, 2007: 171-180.

[25] Sels V. and Vanhoucke M. A Hybrid Dual-Population Genetic Algorithm for the Single Machine Maximum Lateness Problem. Evolutionary Computation in Combinatorial Optimization, Lecture Notes in Computer Science, 2011, Volume 6622: 14-25.

[26] http://people.sc.fsu.edu/ jburkardt/datasets/knapsack_01/ p07_c.txt, 12th August, 2013.

[27] https://github.com/jkdevey ra/CM SC-170-Knap sack GA/tree/master/src/main/java/dataset, 12th August, 2013.

\section{Authors' Profiles}

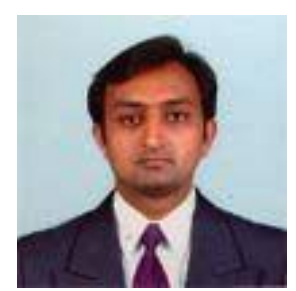

A. J. Umbarkar is presently working as an Assistant Professor in Information Technology at Walchand College of Engineering, at Sangli, MS, India. He has received his Bachelor of Engineering (BE) in Computer Engineering from PICT, Pune, MS, India and his Master of Engineering (ME) from Computer Science and Engineering (CSE) from Walchand College of Engineering, at Sangli, MS, India.

$\mathrm{He}$ is perusing $\mathrm{PhD}$ from Government College En gineering, Aurangabad, from University: BAMU Aurangabad, MS, India. He has 12 years of teaching experience at UG and 6 years at PG. His research interests include Parallel Genetic Algorithms, Parallel Evolutionary Algorithms and Parallel programming. He has published about 16 research papers in Conferences and Journals.

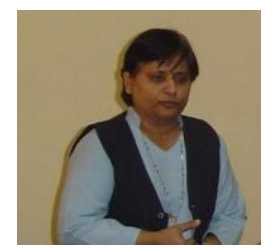

Madhuri S. Joshi is presently working as a Professor, Department of Computer Engineering, Jawaharlal Nehru Engineering College, Aurangabad, M.S., India. She received her B.E. (Electronics $\&$ Telecom) from College of En gineering, Pune (1985), M. Tech. (CS) from IIT Madras (1993) and Ph.D. in 2008. Her research interests are Image Processing, Pattern Recognition, Data Mining, Operating Systems. She has published about 25 research papers in Conferences and Journals.

How to cite this paper: A. J. Umbarkar, M. S. Joshi,"0/1 Knapsack Problem using Diversity based Dual Population Genetic Algorithm", International Journal of Intelligent Sy stems and Applications(IJISA), vol.6, no.10, pp.34-40, 2014. DOI: 10.5815/ijisa.2014.10.05 